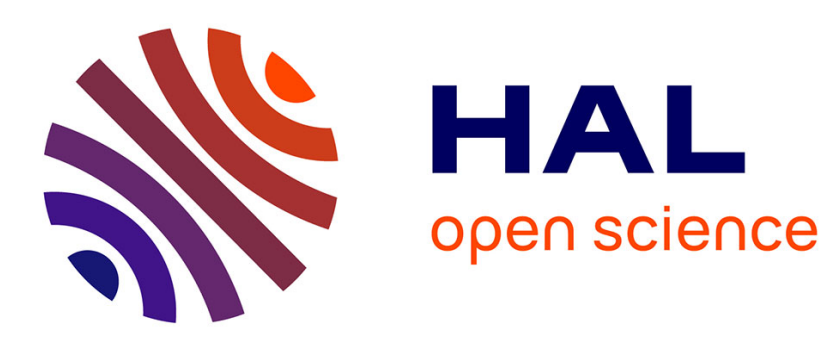

\title{
Scattering model of a cylindrical shell with internal axisymmetric frames by using the circumferential admittance approach \\ Laurent Maxit
}

\section{- To cite this version:}

Laurent Maxit. Scattering model of a cylindrical shell with internal axisymmetric frames by using the circumferential admittance approach. Applied Acoustics, 2014, 80, pp.10-22. 10.1016/j.apacoust.2014.01.002 . hal-00994286

\section{HAL Id: hal-00994286 \\ https://hal.science/hal-00994286}

Submitted on 10 Jun 2015

HAL is a multi-disciplinary open access archive for the deposit and dissemination of scientific research documents, whether they are published or not. The documents may come from teaching and research institutions in France or abroad, or from public or private research centers.
L'archive ouverte pluridisciplinaire HAL, est destinée au dépôt et à la diffusion de documents scientifiques de niveau recherche, publiés ou non, émanant des établissements d'enseignement et de recherche français ou étrangers, des laboratoires publics ou privés. 


\title{
Scattering model of a cylindrical shell with internal axisymmetric frames by using the circumferential admittance approach
}

\author{
Laurent MAXIT \\ INSA Lyon, Laboratoire Vibrations-Acoustique (LVA), \\ 25 bis, av. Jean Capelle \\ 69621 Villeurbanne, France \\ laurent.maxit@insa-lyon.fr
}

\begin{abstract}
A numerical model is proposed for predicting scattering pressure by a fluid-loaded cylindrical shell stiffened by axisymmetric internal frames and impacted by an acoustic plane wave. The proposed developments are based on the circumferential admittance approach (CAA) which allows us assembling a numerical model of the fluid loaded shell with finite element models of the internal frames. The scattering pressure model deduced with the CAA can then take into account: (a), internal frames having a cross section with a complex geometry and thickness variations (like T-shaped stiffeners, bulkheads, hemispherical end caps); (b), variations of frame spacings; (c), frame-shell coupling in the three translational directions and tangential rotation. Comparison with the numerical and experimental results of the literature for a periodic stiffened shell shows that the scattering from Bragg, Bloch-Floquet, and Helical waves is correctly predicted. The effects on the backscattering pressure of axial and tangential coupling forces are highlighted. Finally, an example of a non-periodically stiffened shell is presented to highlight the versatility of the approach proposed.
\end{abstract}




\section{Introduction}

The scattering of an acoustic plane wave by cylindrical shells has been the subject of numerous works. Under the pressure loading of a harmonic plane wave, an elastic shell vibrates and re-radiates noise, adding to the specular reflection and geometric diffraction of the incident wave., Certain mechanical and geometrical properties of the shell can be estimated by analysing this radiated pressure due to shell deformation. Different types of shells have been studied in the past: infinite cylindrical shells [1,2] were modelled by using the elastic theory. The phase matching of shell helical waves with obliquely incident waves was highlighted and seen to have a significant influence on backscattered pressure. This was confirmed experimentally in [2]. It was shown that the compressional and shear helical waves are the main contributors to the scattered field in the frequency domain. The attenuation of these waves by using a viscoelastic layer was studied in [3]. The scattering from finite cylindrical shells was studied by different authors [4-6]. An asymptotic solution of scattering was obtained in [4] by considering that the cylindrical shell is slender and that its ends are simply supported. Scattered pressure due to the elastic deformation of the finite shell was compared to rigid shell scattering for monostatic and bistatic cases. Measurements of the backscattering pressure from an insonified finite cylindrical shell immersed in water were proposed in [5]. The frequency band of investigation corresponded to $5<k a<15$ and the effects of membrane waves were studied. The backscattering pattern in the frequency- angle domain presented loci along which exist discrete areas that represent high levels of backscattering energy. The positions of the loci were easily associated with the membrane circumferential modes of an infinite shell. Comparison with an approximate theoretical model permitted associating the position of the high level spots to the resonances (i.e. axial and circumferential) of the finite shell. Measurements at higher frequencies were carried out in [7], showing the effect of flexural waves on the resulting loci for a thick finite cylindrical shell. A ray theory was proposed in [8] which allowed predicting the increased backscattering observed experimentally for the membrane and flexural waves. Shells of different lengths were investigated experimentally in [6]. A large frequency band corresponding to $10<k a<50$ was considered to highlight the effects of both membrane and flexural waves. The scattering of a periodically ribbed finite cylindrical shell has been studied over the last two decades. Monostatic measurements in the domain $2<k a<30$ on two cylindrical shells, one stiffened with regularly spaced ring stiffeners and the other without stiffeners, highlighted the effect of 
periodic discontinuities [9]. Bragg scattering and scattering from Bloch-Floque waves were observed in the mid-frequency domain: the first phenomenon was due to the interference of the geometrical reflexions of the incident plane waves on the ring stiffeners whereas as the second one results from the propagation of flexural waves interacting with the periodic stiffeners $[10,11]$. These phenomena were found to be more dominant than those associated with the scattering from the compressional and shear helical waves mentioned previously. Models for evaluating the flexural dispersion curves of the ribbed shell were proposed. They permit interpreting the effect of the Bloch-Floquet waves of the scattering and approximating the location of the highlights on the experimental scattering patterns. A model of the scattering of an obliquely incident wave by a ribbed finite cylindrical shell was proposed in [12]. The shell was assumed to be slender, described by a thin shell theory (i.e. Donnel equations) and stiffened by a set of periodically spaced rings. Only the normal component of the reactive forces exerted by the stiffeners on the shell was considered. Backscattering pressures calculated with this model were in agreement with measurements published previously [13]. A comparison with new measurements was proposed in $[10,11]$ and an simple models were used to interpret the different phenomena. The model proposed in [12] was improved for dealing with a shell with irregularly spaced ribs [14]. The ribs, which could be coupled with axisymmetric oscillators, were described by their modal impedances and only the normal component of the reactive forces was considered. The backscattered pressure was approximated by selecting the contribution of the axial modes approximately satisfying the spatial coincidence condition. This model was used to study the influence of small aperiodicity and axisymmetric oscillators.

In a previous paper by the present author [15], the Circumferential Admittance Approach (CAA) was presented for predicting the vibration and pressure radiated from a submerged cylindrical shell stiffened non periodically by axisymmetric internal frames. The external excitations considered in [15] were mechanical point forces on the internal frames. The $(k, M)$ reciprocity technique proposed in [16] also permits use the CAA for a shell excited by the pressure fluctuations induced by a homogeneous turbulent boundary layer developed at the shell surface. In the present paper, we propose an extension of the CAA model [15] to estimate the reradiated pressure of the stiffened shell impacted by an obliquely incident plane wave. This extension could be used for studying the backscattering pressure due to the elastic deformation of the shell and the internal frames. Basically, the CAA consists in substructuring the problem: the immerged cylindrical shell is considered on one part and the internal frames 
on the other parts. Circumferential admittances are estimated with a numerical model for the cylindrical shell and with the Finite Element Method (FEM) for the internal frames. The assembly of these admittances makes it possible to calculate the coupling forces. In the second step, the forces are injected into the numerical model of the fluid-loaded shell to calculate its vibrations and radiated noise.

With the CAA model, the internal frames can be irregularly spaced. As the frames are modelled with FEM, it is easy to then consider different frame geometries: stiffeners with I or T-shaped sections, bulkheads with flat or curve sections, etc. Moreover, the three components of reacting forces and the tangential reacting moment between the frames and the shell are taken into account. The model developed in this paper could be used in the future for studying the influence of these different parameters on the backscattering pressure. In particular, it will permit considering bulkheads that can lead to significant changes to the scattering of compressional and shear helical waves due to axial and tangential coupling forces, as highlighted for a specific case in [17-19]. We can emphasize that the circumferential admittances approach is based on the same principle than the mobility matrix approach considered in [18-19] (sometimes called receptance, mobility or impedance approaches). However, in [18-19], it was developed for studying only the acoustic scattered pressure from a fluid-loaded cylindrical shell with one or two internal plate bulkheads, for the low circumferential mode order equals 0 and 2. In the present paper, the use of FEM for modelling the internal frames would permit to study the effect of the design of the bulkheads (curvatures, thickness variation, etc). Moreover, the effect on the acoustic scattering of the interaction between the bulkheads and the periodic ring stiffeners could also be studied. The dramatic increase of computer capabilities since 1995 and the use of the spectral approach for evaluating the shell admittances [15] allow us to consider well higher circumferential modes than in [18-19]. The interaction of the flexural waves with the internal frames can then be studied in the present paper. It was not the case in [18-19] which was limited to the interaction of the compressional and shear helical waves (with low-order circumferential modes).

The present paper is organized as follows:

- After the presentation of the problem considered, the main steps of the circumferential admittance approach are described in Sec. III; 
- Section IV consists of the analytical calculation of the displacements of the cylindrical shell (without the frames) when it is excited by an obliquely incident plane wave. These displacements characterise the external excitation in the CAA;

- The process to estimate the radiated pressure of the shell stiffened by its internal frames with the CAA is proposed in Sec. V;

- Section VI consists in comparing the CAA results with results from the literature for a periodically stiffened shell;

- Before concluding, an illustration of the present approach is proposed in Sec. VII by considering a shell composed of two parts with two different rib spacings.

\section{Presentation of the problem}

\section{FIGURE 1}

Let us consider the ring-stiffened cylindrical shell immersed in water shown in Fig. 1. The cylindrical coordinates $(x, r, \theta)$ are considered for describing this system where $x$ is the axial coordinate, $r$ the radial coordinate, and $\theta$ the circumferential angular coordinate. The external fluid has mass density $\rho_{0}$ and acoustic wave speed $c_{0}$.

The shell is impacted by an acoustic harmonic plane wave of oblique incidence compared to the axial direction of the shell. For the sake of simplicity, the wavevector of this incidence wave is assumed to be contained in the plane $\theta=0$ and it forms an angle $\xi^{i}$ with the normal of the axial axis (see Fig. 1). The time dependence of this harmonic excitation is $e^{j \omega t}$ for which $\omega$ is the angular frequency. Considering the stationary response of the system, the time dependence $e^{j \omega t}$ for the excitation and response will be omitted in the notation.

The incident pressure of amplitude $p_{0}$ is given by

$$
p^{i}(x, r, \theta)=p_{0} e^{j \bar{k}_{x} x+j \bar{k}_{r} r \cos \theta}
$$

with $\bar{k}_{x}=k_{0} \sin \xi_{i}, \bar{k}_{r}=k_{0} \cos \xi_{i}$ and $k_{0}$, the acoustic wavenumber (i.e. $k_{0}=\omega / c_{0}$ ). 
The $\Theta$ internal frames are composed of different types of stiffeners, bulkheads, hemispherical endcaps, etc. These frames are assumed to be axially symmetric and located at axial coordinate $x_{i}, i \in[1, \Theta]$. The connections between the shell and the frames are rigid. The lines of attachment are circles of radius $R$, the mean radius of the cylindrical shell. The materials of the shell and frames are linearly elastic, homogeneous and isotropic. $\rho, E, v$ are, respectively, mass density, Young modulus $E$, and Poisson ratio.

As required by the CAA, the cylindrical shell is assumed to be of constant thickness $h$ and of infinite length. In order to simulate shells of finite length, boundary conditions can be introduced by introducing fictive frames with specific admittances (see [15]). The part of the shell between the two extreme frames (i.e. $x \in\left[x_{1}, x_{\Theta}\right]$ ) is called the "active part". The rest of the shell can be considered as representing the boundary conditions for the shell and the fluid medium. We focus on estimating the pressure reradiated by the "active part" of this shell when impacted by the obliquely incident wave. To this end, the circumferential admittance approach described in [15] is used. We recall the different steps of the calculation in the next section.

\section{Principle of the CAA}

\section{- Partitioning}

As shown in Fig. 2, the system considered is partitioned such that the fluid loaded shell constitutes one subsystem and the frames constitute other independent subsystems. We define the variables for the coupling forces and the displacements at the junction between the shell and the $i^{\text {th }}$ frame force:

- $W_{i}^{\text {shell }}, V_{i}^{\text {shell }}, U_{i}^{\text {shell }}$, and $\varphi_{i}^{\text {shell }}$ are the radial / tangential / axial displacements, and the tangential rotation $(\varphi=\partial W / \partial x)$ of the shell, respectively;

- $F_{i}^{\text {shell }}, T_{i}^{\text {shell }}, L_{i}^{\text {shell }}$ and $M_{i}^{\text {shell }}$ are the radial / tangential / axial forces, and the tangential moment exerted on the shell, respectively. 
The same quantities (noted with the exponent "frame" instead of "shell") are defined for the $i^{\text {th }}$ frame.

These variables are a function of the circumferential angle, $\theta$. Taking the $2 \pi$ periodicity into account, they can be decomposed into a Fourier series which is written for the variable $f$,

$$
f(\theta)=\sum_{n=-\infty}^{\infty} \tilde{f}_{n} e^{j n \theta} \text { with } \tilde{f}_{n}=\frac{1}{2 \pi} \int_{0}^{2 \pi} f(\theta) e^{-j n \theta} d \theta,
$$

where $n$ is the circumferential order and $\tilde{f}_{n}$, the associated circumferential amplitude.

As $\theta=0$ is a symmetric plane of the system considered, only the integer of the circumferential order will be considered (because $\left|\tilde{f}_{n}\right|=\left|\tilde{f}_{-n}\right|$ ). In the following step, this circumferential order will be omitted in the notation although it will be considered hereafter for variables written with the tilde symbol.

\section{FIGURE 2}

\section{- Definition of the circumferential admittances and the free displacements}

The circumferential admittances of the shell (respectively frame) between the $i^{\text {th }}$ junction and the $j^{\text {th }}$ junction, $\tilde{Y}_{\xi_{i} \zeta_{j}}^{\text {shell }}$ (respectively $\tilde{Y}_{\xi_{i} \zeta_{j}}^{\text {frame }}$ ) is defined by:

$$
\tilde{Y}_{\xi_{i} \zeta_{j}}^{\text {shell }}=\frac{\tilde{\xi}_{i}^{\text {shell }}}{\tilde{\zeta}_{i}^{\text {shell }}}\left(\text { respectively } \tilde{Y}_{\xi_{i} \zeta_{j}}^{\text {frame }}=\frac{\tilde{\xi}_{i}^{\text {frame }}}{\tilde{\zeta}_{i}^{\text {frame }}}\right),
$$

where $\xi \in\{W, U, V, \varphi\}$ and $\zeta \in\{F, L, T, M\}$.

The shell admittances can be calculated with the spectral method presented in [15]. These numerical calculations are based on the Függe thin shell theory, a resolution of the problem in the wavenumber space and the use of a result obtained for a simplified problem (i.e. shell with the fluid added mass) which allows accelerating the numerical convergence. Moreover, the 
invariance in translation about the axial direction of the problem (i.e. fluid loaded cylindrical shell) can be used to save considerable computation times. This is the reason why a shell of constant thickness and infinite length was considered.

The Finite Element Method can be used to calculate frame admittances. In [15], axisymmetric shell elements and the MD NASTRAN code were considered for these calculations. In the present paper, the calculations were performed with the SDtool FEM code [20]. The frames are represented by a 3-D mesh and shell elements. The Fourier decomposition (2) was then done with a homemade MATLAB code. The comparison between the two methods shows good agreement. The second one is more costly in terms of computation time but it could be used in the future for dealing with non axisymmetric frames. However, it is not the subject of the present paper.

The external excitation is characterised in CAA by the free displacement of the shell, $\tilde{\bar{\xi}}_{i}^{\text {shell }}$. This corresponds to the displacements of the fluid loaded cylindrical shell (without the frames) impacted by the incident acoustic waves. The calculations of these quantities will be given in section IV.

\section{- Assembling and calculating the coupling force}

The coupling between the fluid-loaded shell and the internal frames is performed in two steps:

- the first step consists in using the linearity properties of the system to express the relationship at the junctions between the displacements and the forces for the shell and the frames, separately;

- the second step consists in writing the continuity conditions at each junction, namely the force equilibrium and the equality of displacement. 
We finally obtain the linear matrix system:

$$
\left[\tilde{\mathrm{Y}}^{\text {shell }}+\tilde{\mathrm{Y}}^{\text {frame }}\right] \tilde{\mathrm{F}}^{\text {frame }}=-\tilde{\overline{\mathrm{W}}}^{\text {shell }},
$$

where:

- $\tilde{\mathrm{Y}}^{\text {shell }}$ and $\tilde{\mathrm{Y}}^{\text {frame }}$ are matrices containing the shell and frame admittances, respectively;

- $\tilde{\mathrm{F}}^{\text {frame }}$ is a vector containing the coupling forces exerted by the frames on the shell;

- $\tilde{\overline{\mathrm{W}}}^{\text {frame }}$ is a vector containing the free displacements of the shell.

These matrices / vectors are built by ordering the associated quantities as a function of the order of the junctions, $i$ and the 4 degrees of freedom (i.e. 3 translations +1 rotation). The vectors have $4 \Theta \times 1$ as dimensions whereas those of the matrices are $4 \Theta \times 4 \Theta$.

By solving this system, we deduce the forces and the moments exerted by the frames on the shell when they are coupled together. In the second step, as described in Sec. V further on, these reaction forces and moments will be re-injected in the cylindrical shell model to deduce the pressure reradiated by the shell coupled with its internal frames. Before that, in Sec. IV we give the developments of the calculation of the right member of Eq. (4), namely the free displacements of the cylindrical shell.

\section{Free displacements of the immerged shell for an oblique incident wave}

In this section, the displacements of the cylindrical shell (without its internal frames) are calculated when it is impacted by an oblique incident wave.

When dealing with a scattering problem [21], the standard method consists in decomposing the total pressure in the fluid medium as the sum of the blocked pressure, $p^{b}$ and the reradiated pressure, $p^{r}$ : 


$$
p=p^{b}+p^{r}
$$

The blocked pressure corresponds to the pressure in the fluid when the shell impacted by the incident wave is considered as rigid. It satisfies:

(E1), the Helmholtz equation with a source term representing the incident wave in the fluid domain;

(E2), the Euler equation with a Neumann condition at the shell surface;

(E3), the Sommerfeld radiation condition at the infinite boundary.

This blocked pressure is used in the second step to introduce the excitation in the problem of the elastic shell interacting with the fluid domain. Indeed, the difference between the total pressure and the blocked pressure gives us the so called reradiated (or scattered) pressure, $p^{r}$. It satisfies:

(E4), the homogeneous Helmholtz equation in the fluid domain;

(E5), the Euler equation depending on the radial shell velocity at the shell surface; (E6), Sommerfeld radiation condition at the infinite boundary.

In the equation of motions of the shell, the pressure distribution acting on the shell appears in the right member and can be decomposed with (5). Then, the blocked pressure $p^{b}$ defined from (E1-E3) can be interpreted as the external excitation of the shell, whereas the reradiated pressure $p^{r}$ defined by (E4-E6) can be interpreted as the fluid reaction due to the shell vibrations. After determining the blocked pressure, $p^{b}$ in Sec. 4.1, the shell displacement can be calculated in Sec. 4.2 from the shell equations of motion and the fluid equations (E4-E6).

\subsection{Blocked pressure}

(E1-E3) define the blocked pressure $p^{b}$. This term can also be decomposed as the sum of the incident pressure $p^{i}$ and the scattered pressure by the rigid shell, $p^{s}$ :

$$
p^{b}=p^{i}+p^{s}
$$


The incident pressure of the oblique plane wave (1) can be expressed as a function of the cylindrical harmonics [21]:

$$
p^{i}(x, r, \theta)=e^{j \bar{k}_{x} x} \sum_{n=-\infty}^{\infty} \tilde{p}_{n}^{i}(r) e^{j n \theta}
$$

with,

$$
\tilde{p}_{n}^{i}(r)=p_{0} j^{n} J_{n}\left(\bar{k}_{r} r\right)
$$

$J_{n}$ is the Bessel function of first kind of order $n$.

The scattered pressure $p^{s}$ (i.e. $p^{s}=p^{b}-p^{i}$ ) verifies the homogeneous Helmholtz equation, the Sommerfeld radiation condition and the Euler equation with the Neumann condition, giving:

$$
\frac{\partial p^{s}}{\partial r}(x, R, \theta)=-\frac{\partial p^{i}}{\partial r}(x, R, \theta)
$$

The solutions of the homogeneous Helmholtz equation respecting the Sommerfeld condition can be expressed in cylindrical harmonics. The scattered pressure, $p^{s}$ can be determined by introducing these solutions and the incident pressure decomposition (7-8) in (9), Finally, the blocked pressure can be deduced with (6):

$$
\begin{gathered}
p^{b}(x, r, \theta)=e^{j \bar{k}_{x} x} \sum_{n=-\infty}^{\infty} \tilde{p}_{n}^{b}(r) e^{j n \theta}, \text { with } \\
\tilde{p}_{n}^{b}(r)=p_{0} j^{n}\left[J_{n}\left(\bar{k}_{r} r\right)-\frac{1}{\cos \xi_{i}} \frac{J_{n}^{\prime}\left(\bar{k}_{r} R\right)}{H_{n}^{(2))^{\prime}}\left(\bar{k}_{r} R\right)} H_{n}^{(2)}\left(\bar{k}_{r} r\right)\right],
\end{gathered}
$$

where $H_{n}^{(2)}$ is the Hankel function of the second kind of order $n$. The prime symbol indicates the derivative with respect to the argument of the function considered (i.e. Bessel or Hankel function).

\subsection{Shell displacement}

The shell displacements can be calculated by using the developments described in Sec. 4.A. of [15]. The motions of the shell are represented by the Flugge equation. With the Fourier transform definition, 


$$
\tilde{f}(x) \stackrel{F . T .}{\longrightarrow} \tilde{f}\left(k_{x}\right)=\int_{-\infty}^{\infty} f(x) e^{-j k_{x} x} d x
$$

the spectral displacements of the shell $\tilde{\tilde{U}}, \tilde{\tilde{V}}, \tilde{\tilde{W}}$, can be written in the wavenumber space

$$
\tilde{\tilde{U}}=\frac{\tilde{\tilde{p}}^{b}}{\tilde{\tilde{\Delta}}^{\prime}}\left(\tilde{\tilde{Z}}_{U V} \tilde{\tilde{Z}}_{V W}-\tilde{\tilde{Z}}_{V V} \tilde{\tilde{Z}}_{U W}\right), \tilde{\tilde{V}}=\frac{\tilde{\tilde{\tilde{p}}}^{b}}{\tilde{\tilde{\Delta}}^{\prime}}\left(\tilde{\tilde{Z}}_{U W} \tilde{\tilde{Z}}_{U V}-\tilde{\tilde{Z}}_{U U} \tilde{\tilde{Z}}_{V W}\right), \tilde{\tilde{W}}=\frac{\tilde{\tilde{p}}_{b}}{\tilde{\tilde{\Delta}}^{\prime}}\left(\tilde{\tilde{Z}}_{U U} \tilde{\tilde{Z}}_{V V}-\tilde{\tilde{Z}}_{U V}{ }^{2}\right),
$$

with $\tilde{\tilde{\Delta}}^{\prime}=-\frac{2 \pi E}{h\left(1-v^{2}\right) R^{2}} \tilde{\tilde{\Delta}}$.

Analytical expressions of $\tilde{\tilde{Z}}$ and $\tilde{\tilde{\Delta}}$ are given in [15]. These quantities depend on the wavenumber $k_{x}$, the circumferential order $n$ and the geometrical and mechanical properties of the shell.

The spectral blocked pressure $\tilde{\tilde{p}}_{b}$ is expressed from (10):

$$
\tilde{\tilde{p}}_{n}^{b}\left(k_{x}\right)=2 \pi \delta\left(k_{x}-\bar{k}_{x}\right) \tilde{p}_{n}^{b}(R)
$$

where $\delta$ is the Dirac delta function.

The shell displacement can then be written along the $\mathrm{x}$-axis by an inverse Fourier transform:

$$
\begin{aligned}
& \tilde{\bar{U}}_{n}(x)=\frac{\tilde{p}_{n}^{b} e^{j \bar{k}_{x} x}}{\tilde{\tilde{\Delta}}^{\prime}\left(\bar{k}_{x}, n\right)}\left[\tilde{\tilde{Z}}_{U V}\left(\bar{k}_{x}, n\right) \tilde{\tilde{Z}}_{V W}\left(\bar{k}_{x}, n\right)-\tilde{\tilde{Z}}_{V V}\left(\bar{k}_{x}, n\right) \tilde{\tilde{Z}}_{U W}\left(\bar{k}_{x}, n\right)\right], \\
& \tilde{\bar{V}}_{n}(x)=\frac{\tilde{p}_{n}^{b} e^{j \bar{k}_{x} x}}{\tilde{\tilde{\Delta}}^{\prime}\left(\bar{k}_{x}, n\right)}\left[\tilde{\tilde{Z}}_{U W}\left(\bar{k}_{x}, n\right) \tilde{\tilde{Z}}_{U V}\left(\bar{k}_{x}, n\right)-\tilde{\tilde{Z}}_{U U}\left(\bar{k}_{x}, n\right) \tilde{\tilde{Z}}_{V W}\left(\bar{k}_{x}, n\right)\right], \\
& \tilde{\overline{\mathrm{W}}}_{n}(x)=\frac{\tilde{p}_{n}^{b} e^{j \bar{k}_{x} x}}{\tilde{\tilde{\Delta}}^{\prime}\left(\bar{k}_{x}, n\right)}\left[\tilde{\tilde{Z}}_{U U}\left(\bar{k}_{x}, n\right) \tilde{\tilde{Z}}_{V V}\left(\bar{k}_{x}, n\right)-\tilde{\tilde{Z}}_{U V}^{2}\left(\bar{k}_{x}, n\right)\right] .
\end{aligned}
$$

The free displacements of the shell which intervenes in the right member of (4) can finally be deduced from the previous displacement expressions evaluated at each junction position on the axial axis:

$$
\tilde{\bar{U}}_{i}^{\text {shell }}=\tilde{\bar{U}}_{n}\left(x_{i}\right), \tilde{\bar{V}}_{i}^{\text {shell }}=\tilde{\bar{V}}_{n}\left(x_{i}\right), \tilde{\bar{W}}_{i}^{\text {shell }}=\tilde{\bar{W}}_{n}\left(x_{i}\right), \tilde{\bar{\phi}}_{i}^{\text {shell }}=-j \bar{k}_{x} \tilde{\bar{W}}_{n}\left(x_{i}\right), \forall i \in[1, \Theta]
$$

For a given incident angle $\xi_{i}$ (i.e. $\bar{k}_{x}$ and $\bar{k}_{r}$ fixed), the free displacements can be calculated with little computing time using (15-16). Then, the matrix system (4) allows calculating the coupling forces exerted by the frames on the shell when they are coupled together. In Sec. V, 
these forces will be introduced in the cylindrical shell model to estimate the reradiated pressure in the far field.

\section{Reradiated pressure from the stiffened shell}

This section consists in the calculation of the reradiated pressure by the cylindrical shell coupled to its internal frames when it is impacted by an obliquely incident wave. We recall that the reradiated pressure is defined as the complement of the blocked pressure to give the total pressure in the fluid medium (see equation (6)). It does not take the specular and geometric scattering of the rigid body into account. This is not dealt with, however, in the present paper as these phenomena are not influenced by the internal frames and the elastic deformation of the shell. On the other hand, as the cylindrical shell is assumed infinite for the sake of convenience in the CAA, we focus on estimating the reradiated pressure from the part previously called the "active part" which corresponds to the truncated shell between the two extreme frames.

The calculation is then decomposed into three steps: first, the spectral displacements of the framed shell are calculated with the spectral method given in [15]; second, the spectral displacements of the "active part" are estimated by spatial windowing; lastly, the reradiated pressure is estimated in the far field by using the stationary phase theorem.

\subsection{Spectral displacement of the framed shell}

When the shell is coupled with its internal frames and impacted by an acoustic plane wave, the pressure distributions exerted on the fluid loaded cylindrical shell are due to the line forces exerted by the frames on the shell, the blocked pressure and the reradiated pressure. In the mathematical formulation, they intervene in the right member of the motion equations of the shell. The reradiated pressure respects the homogeneous Helmholtz equation, the Sommerfeld condition and the Euler equation links it to the radial velocity of the shell. The problem is solved in the wavenumber space by again calling on the spectral method [15] In the present case, the spectral pressure distributions can be written as:

$$
\left\{\begin{array}{l}
\tilde{\tilde{p}}_{U U}=\sum_{i=1}^{\Theta} e^{-j k_{x} x_{i}} \tilde{L}_{i}^{\text {frame }}, \\
\tilde{\tilde{p}}_{V V}=\sum_{i=1}^{\Theta} e^{-j k_{x} x_{i}} \tilde{T}_{i}^{\text {frame }}, \\
\tilde{\tilde{p}}_{W W}=\tilde{\tilde{p}}^{b}-\sum_{i=1}^{\Theta} e^{-j k_{x} x_{i}}\left(\tilde{F}_{i}^{\text {frame }}+j k_{x} \tilde{M}_{i}^{\text {frame }}\right) .
\end{array}\right.
$$


One deduces the radial spectral displacements of the framed shell [15]:

$$
\tilde{\tilde{W}}=\frac{\tilde{\tilde{p}}_{U U}\left(\tilde{\tilde{Z}}_{U V} \tilde{\tilde{Z}}_{V W}-\tilde{\tilde{Z}}_{U W} \tilde{\tilde{Z}}_{V V}\right)+\tilde{\tilde{p}}_{V V}\left(\tilde{\tilde{Z}}_{U W} \tilde{\tilde{Z}}_{U V}-\tilde{\tilde{Z}}_{V W} \tilde{\tilde{Z}}_{U U}\right)+\tilde{\tilde{p}}_{W W}\left(\tilde{\tilde{Z}}_{U U} \tilde{\tilde{Z}}_{V V}-\tilde{\tilde{Z}}_{U V}{ }^{2}\right)}{\tilde{\tilde{\Delta}}^{\prime}} .
$$

This formula gives us the radial displacements as a function of the axial wavenumber $k_{x}$ and the circumferential order.

\subsection{Spectral displacements of the active part of the framed shell}

The spectral displacements of the "active part" are deduced from (18) by applying space windowing. To do this, an inverse Fourier transform of the spectral displacements is considered to express them as a function of the axial coordinate $x$. Consequently, this transform cannot be obtained analytically but is deduced numerically.

The wavenumber space $k_{x}$ is truncated with a sampling wavenumber $\bar{k}_{x}$ (i.e. $k_{x} \in\left[-\bar{k}_{x}, \bar{k}_{x}\right]$ ) and sampled with a wavenumber resolution $\delta k_{x}$. In order to avoid aliasing and loss of information, the criteria for defining these parameters were given in [15] (see Eq. (26-27) in this reference). These criteria are considered in the following.

An Inverse Discrete Fourier Transform (IDFT) of the spectral displacements $\tilde{\tilde{W}}$ expressed in the discretized wavenumber space gives us the displacements $\tilde{W}$ as a function of the discrete values of the axial coordinate $x$ :

$$
\tilde{W}\left(k_{x}\right) \stackrel{I D T F}{\longrightarrow} \tilde{W}(x) .
$$

In order to extract the displacements corresponding to the "active part" (i.e. $x \in\left[x_{1}, x_{\Theta}\right]$ ), a Tukey function is considered:

$$
\forall x \in \square, \bar{\Pi}(x)= \begin{cases}\frac{1}{2}\left[1-\cos \left(\frac{\pi\left(x-x_{1}\right)}{\alpha}\right)\right], & \text { if } x \in\left[x_{1}, x_{1}+\alpha\right], \\ 1, & \text { if } x \in\left[x_{1}+\alpha, x_{\Theta}-\alpha\right], \\ \frac{1}{2}\left[1-\cos \left(\frac{\pi\left(x-x_{\Theta}+2 \alpha\right)}{\alpha}\right)\right], & \text { if } x \in\left[x_{\Theta}-\alpha, x_{\Theta}\right], \\ 0, & \text { otherwise, }\end{cases}
$$

where $\alpha$ is the Tukey coefficient. At $\alpha=0, \bar{\Pi}(x)$ becomes rectangular, and at $\alpha=1$ it becomes a Hann window. 
The windowed displacements $\tilde{W}^{w}(x)$ are then defined by:

$$
\tilde{W}^{w}(x)=\tilde{W}(x) \bar{\Pi}(x) .
$$

The Tukey coefficient can be used to avoid strong discontinuities of the windowed displacements. In practice, if the ends of the "active part" are clamped (by using fictive frames with null admittances) or they are stiffened by thick bulkheads, the radial displacements of the shell at the ends (i.e. $\tilde{W}\left(x_{1}\right)$ and $\left.\tilde{W}\left(x_{\Theta}\right)\right)$ are already null or negligible. $\alpha=0$ can be used in this case.

The spectral displacements of the "active part" are finally obtained using the Discrete Fourier Transform (DFT):

$$
\tilde{W}^{w}(x) \stackrel{D T F}{\longrightarrow} \tilde{\tilde{W}}^{w}\left(k_{x}\right) .
$$

Another way for calculating these windowed displacements consists in making a convolution of the original displacements with the Fourier transform of the Tukey function. However, when taking the MATLAB algorithms into account, the process related to (19-22) seems the least time consuming.

\subsection{Reradiated pressure in the far field}

The stationary phase theorem is used to estimate the reradiated pressure in the far field. As illustrated in Fig. 1, an observation point $M$ in the fluid medium defined by $(\mathfrak{R}, \xi, \theta)$ is considered, where $\mathfrak{R}$ is its distance from the origin $\mathrm{O}$ of the cylindrical coordinate system (such that $\mathfrak{R} \gg 2 \pi c_{0} \omega^{-1}$ ), $\xi$ is the angle $\mathrm{OM}$ with the normal of the axial axis and $\theta$ is the circumferential angle related to the cylindrical coordinates. The pressure at this point $M$ reradiated by the "active part" of the frame shell, $p^{w}$ is finally given by the stationary phase theorem:

$$
p^{w}(\Re, \xi, \theta)=\sum_{n=-\infty}^{\infty} \tilde{p}_{n}^{w}(\Re, \xi) e^{j n \theta}
$$

with

$$
\tilde{p}_{n}^{w}(\Re, \xi)=\frac{2 \rho_{0} \omega^{2}}{\Re k_{0} \cos \xi} \frac{\tilde{\tilde{W}}_{n}^{w}\left(-k_{0} \sin \xi\right)}{H_{n}^{(2)^{\prime}}\left(R k_{0} \cos \xi\right)} e^{-j r_{s} k_{0}+j \frac{(n+1) \pi}{2}}
$$


It should be emphasized that only the values of the spectral displacements of the shell $\tilde{W}_{n}^{w}$ for axial wavenumbers $k_{x}$ in the acoustic domain are required (i.e. $k_{x} \in\left[-k_{0}, k_{0}\right]$ ).

The summation of (23) is theoretically infinite. In practice, we will truncate this sum to a maximal circumferential order $\bar{N}$. The choice of this parameter will be discussed for a numerical example in Sec. VI.

On the other hand, as $\theta=0$ is a symmetrical plane of the system considered, the reradiated pressure field has the same property. Since the pressure is a scalar, we obtain $\tilde{p}_{-n}^{w}=\tilde{p}_{n}^{w}$. Eq. (23) can be rewritten as:

$$
p^{w}(\Re, \xi, \theta) \approx \tilde{p}_{0}^{w}(\Re, \xi)+2 \sum_{n=1}^{\bar{N}} \tilde{p}_{n}^{w}(\Re, \xi) \cos (n \theta) .
$$

To conclude the theoretical part of this paper, the different steps of the developed method can be summarized for each circumferential order, $n$ :

- The shell and the frame admittances, $\tilde{\mathrm{Y}}^{\text {shell }}, \tilde{\mathrm{Y}}^{\text {frame }}$, are calculated with the spectral approach [15] and FEM, respectively;

- For a given incident angle, the free displacements of the shell, $\tilde{\overline{\mathrm{W}}}^{\text {shell }}$ are calculated with Eq. (15);

- The forces exerted by the frames on the shell, $\tilde{F}^{\text {frame }}$ are deduced by resolving Eq. $(4)$;

- These coupling forces are injected in the spectral model of the fluid loaded shell to calculate the radial spectral displacements of the framed shell, $\tilde{\tilde{W}}$ with Eq. $(17,18)$;

- The spectral displacements of the "active part" and $\tilde{\tilde{W}}^{w}$ are deduced by the windowing process (19-22);

- The circumferential reradiated pressure in the far field for the incident angle considered, $\tilde{p}_{n}^{w}$, is obtained with Eq. (24).

The reradiated pressure at a given point in the far field, $p^{w}$, is finally given by the sum about the circumferential order of $\tilde{p}_{n}^{w}$ (i.e. Eq. (25)). 


\section{Application to a periodically stiffened shell}

\subsection{Presentation of the test case}

For the first application of the present approach, we consider a periodically stiffened shell previously studied in the literature. The numerical values defining this case are those considered in $[10,11]$. This will allow comparing the results of the present approach with the numerical and experimental ones presented in $[10,11]$.

The finite shell has a length of $750 \mathrm{~mm}$, a radius of $50 \mathrm{~mm}$ and a thickness of $1 \mathrm{~mm}$. It is stiffened by a set of 49 evenly spaced internal rings having a $5 \mathrm{~mm} \times 1 \mathrm{~mm}$ rectangular crosssection. The spacing between two rings is $15 \mathrm{~mm}$. The extremities are closed by $1 \mathrm{~mm}$ thick disks. The shell and the rings are made of steel $\left(E=2.410^{11} \mathrm{~Pa}, \rho=7900 \mathrm{~kg} / \mathrm{m}^{3}, v=0.3\right)$. The shell is immersed in water $\left(c_{0}=1470 \mathrm{~m} / \mathrm{s}, \rho_{0}=1000 \mathrm{~kg} / \mathrm{m}^{3}\right)$. Experimental measurements were performed in a water tank and transducers were used as the emitter and the receiver. The monostatic backscattered pressure as a function of the frequency and the incident angle were obtained after having removed the effect of the specular reflection and the reflections at the two extremities. These results obtained by Liétard et al. (Fig. 5 in [10]) are shown in Fig. 3. The graph is proposed as a function of the non dimensional frequency, $k a$ (i.e. the product of the acoustic wavenumber and the shell radius). High scattering pressure values were linked to different phenomena $[10,11]$ :

- the Bragg scattering due to the interference of the geometrical reflection of the incident plane wave on the rings;

- the helical wave scattering due to the propagation of the shear and compressional helical waves in the shell;

- the scattering of Bloch-Floquet waves resulting from the interaction of the flexural waves with the ribs.

\section{FIGURE 3}

\subsection{Simulation with the approach presented}

Calculations were performed with the CAA. As the damping of the shell and the ring was not characterized in [10], we consider a fixed value of $0.2 \%$ (i.e. $\eta=0.002$ ). We introduced it in the model as a complex Young modulus, $E^{*}\left(\right.$ i.e. $E^{*}=E(1+j \eta)$ ). 
The ring was modelled by FEM using the SDtool code [20] as already mentioned in Sec. III. The values for two ring admittances $-\tilde{Y}_{W F}^{\mathrm{fr}}{ }^{\mathrm{a}}$ (i.e. excitation - reception in the radial direction) and $\tilde{Y}_{\theta M}^{\text {frame }}$ (i.e. excitation - reception in the tangential rotational direction) - are proposed in Fig.4. For each frequency, the first admittances, $\tilde{Y}_{W F}^{\text {frame }}$ exhibit a resonant peak which can be associated with the flexural motions of the ring. A basic 1D beam model allows finding approximately the same result in the low frequency range. The second admittance, $\tilde{Y}_{\theta M}^{\text {frame }}$ exhibits two resonances. They correspond to torsional motions of the ring with deformations of the cross section. This behaviour cannot be reproduced by a 1D rod model with the assumption of a non deformable cross section, thus illustrating one advantage of using the present approach. The complex geometry of the frames (T-shaped stiffener, bulkhead, etc) can be modelled easily, so they can be represented by FEM using shell elements. We emphasize that these calculations should be performed only for each type of frame and they are independent of the frame spacing and the incidence angle.

\section{FIGURE 4}

On another hand, simply supported boundary conditions are simulated at the ends of the "active part" of the shell by inducing two fictive frames with specific admittances. These admittances for the different directions are nulls (i.e. blocked boundary condition) except for the tangential rotation, $\tilde{Y}_{\theta M}^{\text {end }}$ for which a relatively high value allows us to simulate a soft torsional spring. This value was defined in comparison with the shell admittance, $\tilde{Y}_{\theta M}^{\text {end }}$. It was set at 100 times the highest values of these admittances. We underline that the admittances of the end disks can be calculated by FEM and included in the CAA model. However, as the cylindrical shell has the same thickness as the disks and it extends outside the "active part" in the CAA model, it is preferable not to increase the rotational stiffness at the ends by adding the admittances of the end disks.

The shell admittances were calculated with the process described in [15] (including the acceleration convergence method). A numerical study was performed to define a criterion for the maximal circumferential order $\bar{N}$. Fig. 5 illustrates the observations of this study.

\section{FIGURE 5}


The blocked pressure exciting the shell is plotted in Fig. 5a for $k a=30$ as a function of the incidence angle, $\xi_{i}$ and the circumferential order, $n$. A dashed line corresponding to $n_{0}=\operatorname{int}\left[R k_{0} \cos \xi_{i}\right]$ was added to this figure. It can be seen that the blocked pressure decreases quickly when $n$ increases above these values. Only the circumferential orders below or near $n_{0}$ are therefore significantly excited. Fig. 5b-5d shows the implication on the free displacements in the three directions, $\tilde{\bar{U}}_{n}, \tilde{\bar{V}}_{n}, \tilde{\bar{W}}_{n}$. The longitudinal and tangential displacements present significant values only for a small circumferential order, well below $n_{0}$. These orders can be associated with quasi-longitudinal and quasi-shear waves. On the other hand, the radial displacements are significant for values below and around $n_{0}$, as could be expected, but also for orders higher than $n_{0}$. Indeed, a plot of high spot values can be observed for $n$ between 30 and 40. This plot can be associated with the quasi-flexural waves (taking the fluid added mass effect into account). Although the shell is slightly excited on these orders (i.e. low blocked pressure), it can react significantly on its natural flexural motions. This phenomenon occurs only when the excitation frequency approaches the critical frequency associated with the flexural motions (i.e. when $k a=40$ for the present shell). In this case, the orders which can be associated with the flexural waves are relatively close to $n_{0}$. We can then define a criterion for the maximal circumferential order $\bar{N}$ from $n_{0}$ by taking a margin coefficient $\kappa$ large enough to encompass this phenomenon:

$$
\bar{N}=\kappa n_{0} .
$$

Our numerical study shows that $\kappa=2$ is largely enough for the present case. For a damped shell, this value can be slightly reduced.

For the results presented in this section, Eq. (26) is considered and the Tukey coefficient, $\alpha$, defined for windowing the shell displacement in the "active part" (i.e. Eq. (21)) was set at 5 mm. As simply supported boundary conditions were imposed at the ends of the "active part", the radial displacements are negligible at these ends. The backscattering pressure is therefore only slightly influenced by the value of the Tukey coefficient (so it remains small compared to the length of the "active part"). 


\subsection{Results}

Two calculation cases with the present approach were considered:

- The "reduced" case takes only the radial reaction forces between the frames and the shell into account. In this calculation, the frame admittance in the radial direction, $\tilde{Y}_{W F}^{\text {frame }}$, calculated with FEM is considered, whereas large values compared to the shell admittances are prescribed for the other directions of the frame admittances (so simulating very soft springs);

- the "full" case takes into account the reaction forces between the frames and the shell along the four degrees of freedom (i.e. longitudinal, tangential, and radial forces and the tangential moment). In this case, the 16 components of the frame admittance matrix are calculated by FEM.

\subsubsection{Reduced model}

\section{FIGURE 6}

The "reduced" CAA calculation allows comparing our results with that presented in [10] and obtained with the numerical method described in [12]. We emphasize that the model considered in these two references $[10,12]$ does not have exactly the same assumptions as the present one: the rings are represented by a rod of circular curvature and the acoustic field in the fluid is approximated by a continuous distribution of sectorial spherical harmonics adapted for a slender body. However, both models consider only the effect of the radial reaction forces of the rings on the shell. The results of the "reduced" model are proposed in Fig. 6. They can be compared with the numerical results presented in Fig. 6 of Ref. [10]. Good agreement between the two calculations can be observed, especially for the scattering from Bragg and helical waves. The trajectories of the Bloch-Floquet (BF) scatterings are globally similar. It can be noticed, however, that the width of the trajectories is larger with the present approach and two branches appear below a given incidence angle (noted (A) and (B) in Fig. 6). Our results can be explained by analysing the scattering field for different circumferential orders. In order to illustrate the phenomenon involved, we consider the simple scattering/interference model described in [10] for estimating the BF trajectories. The quasiflexural waves at the origin of the Bloch-Floquet scattering can propagate in the shell along 
the both axial and circumferential directions. For a given circumferential order, $n$, the axial decomposition of the flexural wavenumber $k_{x f}(n)$ is

$$
k_{x f}(n)=\sqrt{k_{f}^{2}-\left(\frac{n}{R}\right)^{2}},
$$

where $k_{f}$, is the flexural wavenumber of the equivalent plate taking the fluid loading into account.

\section{FIGURE 7}

Applying the scattering/interference model to axial waves characterized by the wavenumber, $k_{x f}$ gives the BF trajectories shown in Fig. 7 for different circumferential orders (i.e. $n \in[0,5,10,15,20])$. The trajectories are plotted when the condition $k a>\frac{1.2 n}{\cos \xi_{i}}$ is respected, ensuring that the shell is sufficiently excited by the incident wave on the circumferential order considered. Fig. 7 shows that when $n$ increases, the BF trajectories shift to the higher frequencies, beginning from a decreasing incident angle. This phenomenon explains the spreads of the BF trajectories at positions noted (A) and (B) in Fig. 6. However, these trajectories are not spread uniformly. Two branches appear that can be explained by the resonance of the rings. Indeed, Fig. 4 indicates that for $k a$ around 18, the resonance occurs for $n$ around 15. For circumferential orders around this number, the rings do not oppose reaction forces, the flexural waves can propagate freely. BF waves are therefore not generated for these orders. This explains the hole (between the two branches) observed in the BF trajectory at position (A) in Fig. 6. The same analysis allows explaining the two branches at position (B), so the present model gives results in agreement with the different models presented in [10].

Now, let us compare the results of Fig. 6 with the experimental results obtained by Lietard et al and presented in Fig. 3. The scattering from the Bragg, Bloch-Floquet, and helical waves predicted by the model is effectively observed experimentally. The first BF trajectory seems less predominant than those predicted by the "reduced" model. The peak energy (indicated by an asterisk * in Fig. 3) is also noted in our prediction at the intersection between the Bragg and $\mathrm{BF}$ trajectories. 
The simulation with the "reduced" model indicates that the helical waves induce the highest pressure levels while the experimental spectrum does not show significant trajectories for the low incidence angles. It should be emphasized that the results of our predictions are related to the damping loss factor introduced in the model. This dependency on the damping of the scattering from the helical waves had already been observed [3]. Without experimental values, we considered a fixed value of $0.2 \%$. A higher value would reduce the backscattering pressure due to the helical waves.

\subsubsection{Full model}

\section{FIGURE 8}

The results obtained with the "full" CAA model are shown in Fig.8. Before analysing the figure, we underline that the tangential moments exerted by the frames on the shell do not significantly influence the monostatic spectrum for the present case. Indeed, the calculations including the radial forces and the tangential moments between the frames and the shell give practically the same results as in Fig. 6. The comparison of Fig. 8 with Fig. 6 makes it possible to study the effect of the frame reaction forces about the axial and tangential directions.

Three main changes on the spectrum can be observed between the two calculations:

- In the low frequency (noted (I) in Fig. 8), the first BF trajectory no longer appears clearly and the critical angle associated with the helical shear waves increases slightly (i.e. $22^{\circ}$ in Fig. 6 and $27^{\circ}$ in Fig. 8). Moreover, dashed trajectories appear more significantly for $k a \in[10,20]$;

- Additional dashed trajectories (noted (II) in Fig.8) appear for $\xi_{i} \in\left[40^{\circ}, 80^{\circ}\right]$ in the mid frequency;

- Quasi-horizontal patterns (noted (III) in Fig.8) seems to link the Bragg and BF trajectories in the higher frequencies.

It is difficult to explain these modifications at first sight so analysis of the backscattering spectrum for each order was carried out. It should be recalled that without the rings, the coupling of the shear and longitudinal waves with the flexural waves is due to the shell 
curvature and the Poisson effect. Above the ring frequency of the shell (i.e. $k a \approx 4$ ), the effect of the shell curvature vanishes and quasi-flexural waves can propagate. In the presence of the rings, an additional coupling scheme between the different types of waves appears due to the elastic deformations of the rings. The Bloch-Floquet scattering no longer remains related only to the propagation of quasi-flexural waves, but also to the complex coupling of the shear, longitudinal and flexural waves. This coupling of different types of waves could explain why the first BF trajectory corresponding to low circumferential orders virtually disappeared and the dashed trajectories corresponding to higher circumferential orders appeared in the parts noted (I) and (II). It explains also why a slight change of the critical angle associated with the helical shear waves was noticed. It is more difficult to interpret the origin of the quasihorizontal patterns. It appears that they result from interferences between the Bragg and BF scatterings.

The predictions given by the "full" CAA model agree with the experimental ones. The first BF trajectory which appeared clearly with the "reduced" model is found only partially with the "full" model, as with the experiment. The dashed trajectories and the quasi-horizontal patterns were not demonstrated by the experiment. However, these characteristics are of secondary importance for the present case which remains dominated by the scattering of Bragg, helical waves and Bloch-Floquet quasi-flexural waves. The modifications induced by the axial and tangential coupling forces depend on the frame stiffness. For example, bulkheads of higher thickness than the present rings would certainly modify the backscattering spectrums more significantly (as observed in [17] for a shell stiffened by a single bulkhead). Simulations and experiments should be performed in the future on specific shells making it possible to highlight the phenomenon related to the axial and tangential coupling forces.

The different comparisons discussed in Sec. VI reinforce our confidence in the results obtained by the presented approach. In order to illustrate the versatility of the CAA, we propose to apply it to a shell stiffened by two sets of stiffeners in Sec. 7.

\section{Results for a shell with two sets of stiffeners}

The cylindrical shell described previously (i.e. $h=1 \mathrm{~mm}, R=50 \mathrm{~mm}, L=0.75 \mathrm{~m}$ ) is now stiffened by two different types of stiffeners and two different stiffener spacings:

- the first part (i.e. $x \in[0.015 \mathrm{~m}, 0.3 \mathrm{~m}]$ ) is uniformly reinforced by steel stiffeners with a T-cross section of $4 \times 1 \mathrm{~mm}^{2} / 1 \times 3 \mathrm{~mm}^{2}$. The spacing of these stiffeners, $d_{l}$, is set at $15 \mathrm{~mm}$. 
- the second part (i.e. $x \in[0.31 \mathrm{~m}, 0.75 \mathrm{~m}[$ ) is uniformly reinforced by the rings described in Sec. VI. The spacing of the rings, $d_{2}$, is $10 \mathrm{~mm}$.

Although the stiffener spacing is different for the two parts, the shell admittances were obtained with the same numerical process as that used for the periodically stiffened shell of Sec. VI. The admittances of the T-shaped stiffener were calculated by FEM with the SDtool code. It was not necessary to recalculate the admittances of the ring. Indeed, as they were calculated for the case of Sec. VI and saved in a database, it was only necessary to extract them from the database which is one of the advantages of the present approach.

FIGURE 9

FIGURE 10

The results are proposed in Fig. 9. As the shell is more complex than that in Sec. VI (i.e. 2 types of stiffener and 2 spacings), the backscattering spectrum is more complicated than in Fig. 8 and it becomes more difficult to analyse at first sight. In order to identify the Bragg (B) and Bloch-Floquet trajectories (BF), we estimated their positions with the simple scattering/interference model [10] considering the two stiffener spacings. The trajectories estimated for the circumferential order, $n=0$, are plotted in Fig. 10 making it possible to clearly identify in Fig. 9 the Bragg and the low-frequency Bloch-Floquet trajectories due to each part of the shell. A large and chaotic trajectory can be observed in the high frequency. In this frequency range, the simple model presents a superposition of the Bloch-Floquet trajectories of the two parts (due to the relation $2 d_{1}=3 d_{2}$ ). It should be remembered that the trajectories of Fig. 10 were estimated from the flexural wavenumbers. They do not take into account the effect of the frame-shell coupling on the wave propagation in the shell. As the two parts of the shell are not stiffened by the same stiffeners, the superposition of the BF scatterings is not as perfect as predicted in Fig. 10. Moreover, interferences with trajectories of higher circumferential orders (not plotted on Fig. 10) certainly contribute to the assembly of this wide and chaotic trajectory. 
The example shown in this section illustrates the versatility of the approach proposed. This approach can also be used to simulate the presence of bulkheads and hemispherical end caps as small or large variations of the stiffener spacings. In the future, it could be used in parallel with experimental measurements to analyse the scattering of complex stiffened shells.

\section{Conclusions}

This paper proposed the extension of the Circumferential Admittance Approach [15] to obliquely incident plane wave excitations. It allowed us to predict scattering pressure using cylindrical shells stiffened by axisymmetric internal frames. In principle, the method consists in assembling a numerical model of the fluid loaded shell with finite element models of the internal frames. The coupling between the internal frames and the shell is ensured by considering the reaction forces in the three directions and the reaction moment in the tangential direction. The complex geometry of the frame cross-section and thickness variations can be taken into account through the finite element calculations. Thus internal frames like T-shaped stiffeners, curved bulkheads, and hemispherical end caps can be considered in the approach proposed to study their influences on scattering pressure. Moreover, as no assumption is made as to the axial position of the frames, periodically or non-periodically stiffened shells can be considered with this approach.

Results for a periodic stiffened shell were compared with numerical and experimental results taken from the literature showing that the model correctly predicts scattering from Bragg, Bloch-Floquet, and Helical waves. However, calculations including the axial and tangential coupling forces highlighted the influence of these coupling terms on backscattering pressure. They lead to changes in the scattering of the helical waves at low frequencies and the signature of the Bloch-Floquet waves appear more complex than when they are neglected. These changes are, however, of secondary importance for the shell considered.

The versatility of the approach presented was illustrated using a numerical application. The CAA model can be used to study the scattering pressure from a shell stiffened by bulkheads, hemispherical end caps and different types of stiffeners. Small or large variations of the stiffener spacings can also be simulated. In the future, this approach could be used in parallel with experimental measurements to analyse the scattering of a complex stiffened shell. 


\section{Acknowledgments}

This work was performed within the framework of the Labex CeLyA of Université de Lyon, operated by the French National Research Agency (ANR-10-LABX-0060/ ANR-11-IDEX0007).

\section{References}

[1] Flax L, Varadan VK, Varadan VV, Scattering of an obliquely incident acoustic wave by an infinite cylinder, J. Acoust. Soc. Am. 980;68:1832-1835.

[2] Léon F, Lecroq F, Décultot D, Maze G, Scattering of an obliquely incident acoustic wave by an infinite hollow cylindrical shell, J. Acoust. Soc. Am. 1992;91:1388-1397.

[3] Guo YP, Attenuation of helical wave radiation from cylindrical shells by viscoelastic layer, J. Acoust. Soc. Am. 1995;97:298-308.

[4] Tran-Van-Nhieu M, Scattering from a finite cylindrical shell, J. Acoust. Soc. Am. 1992;92:670-679.

[5] Rumerman ML, Contribution of membrane wave reradiation to scattering from finite cylindrical steel shells in water, J. Acoust. Soc. Am. 1993;93:55-65.

[6] Haumesser L, Baillard A, Décultot D, Maze G, Behavior of first guided wave on finite cylindrical shells of various lengths: experimental investigation, J. Acoust. Soc. Am. 2001;109:583-590.

[7] Morse SF, Marston PL, Kaduchak G, High-frequency backscattering enhancements by thick finite cylindrical shells in water at oblique incidence: Experiments, interpretation and calculations, J. Acoust. Soc. Am. 1998;103:785-794.

[8] Morse SF, Marston P, Meridional ray contributions to scattering by tilted cylindrical shells above the coincidence frequency: ray theory and computations, J. Acoust. Soc. Am. 1999;106:2595-2600.

[9] Photiadis DM, Bucaro JA, Houston BH, Scattering from flexural waves on a ribbed cylindrical shell, J. Acoust. Soc. Am. 1994;96:2785-2790.

[10] Lietard R, Influence de raidisseurs internes régulièrement espacés sur la diffusion acoustique par une coque cylindrique finie immergée : diffusion de Bragg et diffusion de Bloch-Floquet (Influence of evenly spaced internal stiffeners on the acoustic scattering by a finite immerged cylindrical shell: Bragg scattering and Bloch-Floquet scattering), Ph. D. dissertation, Université du Havre, Le Havre, 2005. 
[11] Lietard R, Décultot G, Maze G, Tran-Van-Nhieu M, Acoustic scattering from a finite cylindrical shell with evenly spaced stiffeners, J. Acoust. Soc. Am. 2005;118:21422146.

[12] Tran-Van-Nhieu M, Scattering from a ribbed finite cylindrical shell, J.Acoust. Soc. Am. 2001;110:2858-2866.

[13] Houston B, Bucaro JA, Photiadis DM, Broadband acoustic scattering from a ribbed shell, J. Acoust. Soc. Am. 1995;98:2851-2853.

[14] Tran-Van-Nhieu M, Scattering from a ribbed finite cylindrical shell with internal axisymmetric oscillators, J. Acoust. Soc. Am. 2002;112:402-410.

[15] Maxit L, Ginoux JM, Prediction of the vibro-acoustic behavior of a submerged shell non periodically stiffened by internal frames. J. Acoust. Soc. Am. 2010;128:137-151.

[16] Maxit L, Denis V, Prediction of flow induced sound and vibration of periodically stiffened plates, J. Acoust. Soc. Am. 2013;133:146-160.

[17] Guo YP, Sound scattering by bulkheads in cylindrical shells, J. Acoust. Soc. Am. 1994;95:2550-2559.

[18] Cuschieri JM, Feit D, Acoustic scattering from a fluid-loaded cylindrical shell with discontinuities: single plate bulkhead, J. Acoust. Soc. Am. 1995;98:320-338.

[19] Cuschieri JM, Feit D, Acoustic scattering from a fluid-loaded cylindrical shell with discontinuities: double plate bulkhead, J. Acoust. Soc. Am. 1995;98:339-352.

[20] Balmes E, Structural Dynamics Toolbox \& FEMLink, User's Guide (Sdtools, Paris, France, 2012, 696 p.)

[21] Junger MC, Feit D, Sound, structures and their interaction, $2^{\text {nd }}$ ed. (Massachusetts Institute of Technology Press, Cambridge, MA, 1986, 448 p.) 


\section{FIGURE CAPTIONS}

Figure 1. Illustration of a fluid loaded cylindrical shell stiffened by axisymmetric internal frames and impacted by an oblique incident wave.

Figure 2. Illustration of the partitioning.

Upper part, the fluid loaded shell; lower part, the different types of internal frames.

Figure 3. Experimental spectra of backscattered pressure obtained from Ref. [10].

Figure 4. Admittances of the ring: upper part, $\tilde{Y}_{W F}^{\text {frame }}$; lower part, $\tilde{Y}_{\theta M}^{\text {frame }}$.

Figure 5. Values of excitation terms for $k a=30$ (i.e. $f \approx 140 \mathrm{kHz}$ ) as a function of the incidence angle and the circumferential order: (a), blocked pressure, $\tilde{p}_{n}^{b}$ (i.e. Eq. (11)). Free displacements of the shell (i.e. Eq. (15)) in the following directions: (b), longitudinal, $\tilde{\bar{U}}_{n} ;$ (c), tangential, $\tilde{\bar{V}}_{n} ;(\mathrm{d})$, radial, $\tilde{\bar{W}}_{n}$.

Figure 6. Numerical spectrums of monostatic backscattered pressure obtained with the "Reduced" CAA model (i.e. ring-shell coupling through the radial direction only).

Figure 7. Trajectories of Bragg waves (B) and Bloch-Floquet waves for different circumferential orders, $n\left(\mathrm{BF}_{n}\right)$. Calculations with the simple scattering/interference model presented in [10].

Figure 8. Numerical spectrums of monostatic backscattered pressure obtained with the "Full" CAA model (i.e. ring-shell coupling through the 4 degrees of freedom).

Figure 9. Numerical spectra of backscattered pressure of the shell reinforced with T-shaped stiffeners spaced at $15 \mathrm{~mm}$ and rings spaced at $10 \mathrm{~mm}$.

Figure 10. Bragg $\left(\mathrm{B}^{i}\right)$ and Bloch-Floquet $\left(\mathrm{BF}^{i}\right)$ wave trajectories linked to the stiffener spacing, $d_{i}: i=1, d_{1}=15 \mathrm{~mm} ; i=2, d_{2}=10 \mathrm{~mm}$. Calculations for $n=0$ with the scattering/interference model [10]. 